\title{
The CuSPED Mission: CubeSat for GNSS Sounding of the Ionosphere-Plasmasphere Electron Density
}

\author{
Jason N. Gross* ${ }^{*}$ Amy M. Keesee ${ }^{\dagger}$, John A. Christian ${ }^{\ddagger}, \mathrm{Yu} \mathrm{Gu}^{\ddagger}$ and Earl Scime $\S$ \\ West Virginia University, Morgantown, WV, 26506, USA \\ Attila Komjathy 9 \\ California Institute of Technology Jet Propulsion Laboratory, Pasadena, CA, 91011, USA \\ E. Glenn Lightsey ॥ \\ Georgia Institute of Technology, Atlanta, GA, 30332, USA \\ Craig J. Pollock ** \\ NASA Goddard Space Flight Center, Greenbelt, MD, 20771, USA
}

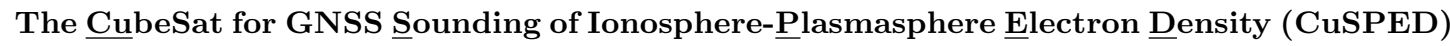
is a $3 \mathrm{U}$ CubeSat mission concept that has been developed in response to the NASA Heliophysics program's decadal science goal of the determining of the dynamics and coupling of the Earth's magnetosphere, ionosphere, and atmosphere and their response to solar and terrestrial inputs. The mission was formulated through a collaboration between West Virginia University, Georgia Tech, NASA GSFC and NASA JPL, and features a 3U CubeSat that hosts both a miniaturized space capable Global Navigation Satellite System (GNSS) receiver for topside atmospheric sounding, along with a Thermal Electron Capped Hemispherical Spectrometer (TECHS) for the purpose of in situ electron precipitation measurements. These two complimentary measurement techniques will provide data for the purpose of constraining ionosphere-magnetosphere coupling models and will also enable studies of the local plasma environment and spacecraft charging; a phenomenon which is known to lead to significant errors in the measurement of low-energy, charged species from instruments aboard spacecraft traversing the ionosphere. This paper will provide an overview of the concept including its science motivation and implementation.
\end{abstract}

Note to reviewers: This CuSPED mission has been proposed and is currently under review. We expect to know the outcome of the proposal's selection prior to AIAA SciTech 2016; and plan to only present this paper if the project is selected and under development at the time of the conference.

\section{Introduction}

Over the past decade, the CubeSat platform and its associated subsystems have evolved from use in purely educational endeavors to being capable of returning high-value science. ${ }^{?}$ Furthermore, the use of GNSS sounding for the measurement of the Earth's ionosphere has led to fundamental discoveries of dynamics of the global ionospheric density. ${ }^{\text {? }}$ The CuSPED mission will leverage both of these important developments to implement a low-cost mission that seeks to meaningfully contribute toward meeting the NASA Heliophysics

\footnotetext{
*Assistant Professor, Department of Mechanical and Aerospace Engineering, AIAA Member

${ }^{\dagger}$ Research Assistant Professor, Department of Physics and Astronomy

$\ddagger^{\ddagger}$ Assistant Professor, Department of Mechanical and Aerospace Engineering, AIAA Senior Member

$\S$ Oleg Jefimenko Professor, Department of Physics and Astronomy

\Senior Member Technical Staff, Ionospheric and Atmospheric Remote Sensing Group

" Professor, Guggenheim School of Aerospace Engineering, AIAA Associate Fellow

**Aerospace Technologist, Fields and Particles
} 
Program decadal science goal ${ }^{\text {? }}$ of determining of the dynamics and coupling of the Earth's magnetosphere, ionosphere, and atmosphere and their response to solar and terrestrial inputs. In particular, a key parameter required for understanding and modeling the coupling of these three regions is the evolution of the plasma density in the ionosphere and lower magnetosphere in response to changing levels of geomagnetic activity. As such, CuSPED will leverage a miniature space-capable GNSS receiver and miniature plasma spectrometer, both of which have heritage from sounding rocket flights, to take important measurements of plasma density in the upper ionosphere and further enable the potential for multi-CubeSat constellations to address this goal.

\section{Mission Science}

\section{A. Particle Precipitation Measurements and Spacecraft Charging}

Low Earth orbit (LEO) missions are ideal for studies of particle populations in the auroral and cusp regions, if such populations can be accurately measured. The pitch angle distribution of low energy ions and electrons are particularly important for models of lower magnetosphere and ionosphere coupling. Such measurements can be combined with ground-based and high altitude measurements to study the interactions between the atmosphere, ionosphere, and magnetosphere. The energization of ionospheric ions during geomagnetic storms drives ion outflow. Oxygen ions with energy $>10 \mathrm{eV}$ are able to escape Earth's gravitational field, thus ion flow in the cusp region can be observed with energies of $10 \mathrm{~s}$ of eV?,? The physics of ion outflow is still poorly understood and is a topic of very active study. Distributed measurements of the low energy plasma populations throughout near-Earth space will provide important inputs to and constraints on theoretical models of the ion outflow process. A multi-platform CubeSat constellation is a cost-effective way that such distributed measurements could be collected.

However, accurate satellite-based plasma measurements are challenging for many reasons, one of which is the charging of the host spacecraft, which can distort measurements, damage instruments, or damage the entire spacecraft. There are various charging mechanisms: immersion in plasma with electron flux greater than ion flux will yield a negative spacecraft potential, while photoemission from solar UV radiation and secondary emission from auroral electron bombardment will yield a positive potential. The net spacecraft potential varies depending on location, the energies and composition of the particle fluxes impacting the spacecraft, the secondary emission properties of exposed spacecraft materials, and the degree of solar illumination.

Low energy ions and electrons become difficult to measure because spacecraft charging distorts the trajectories of these particles. At low enough energies, one charge species is completely repelled from the spacecraft while the other is accelerated to the spacecraft. Thus, it is often impossible to determine anything about the details of the low energy portion of one charge species' population. As noted above, low energy ions play an important role in magnetospheric dynamics by populating the plasma sheet via ion outflow, driving electromagnetic ion cyclotron (EMIC) waves in the magnetosphere, and affecting reconnection rates.? ? Low energy electrons are important for understanding auroral excitation and the acceleration mechanism of ion outflow. ? ? In the ionosphere, very low energy electrons $(<10 \mathrm{eV})$ also drive important chemical processes. The inability to measure low energy populations leaves a significant gap in our understanding of the space plasma environment.

CubeSat missions have less ability to actively control the spacecraft charging and must be able to account for it in their measurements. Large satellite missions that can afford the onboard power resources utilize active control of the spacecraft potential by emitting heavy ions to counter photoemission and eliminate this blind spot in plasma measurement. Other methods to measure low energy particles include placing the instrument on an electrically isolated boom outside of the spacecraft sheath or collecting the repelled particles via the motion of the spacecraft. While booms have been demonstrated on CubeSats, their deployment introduces an additional risk to achieving mission goals.

Scime et al. ${ }^{?}$ modeled the trajectory alteration imposed on low energy electrons measured by positively charged spacecraft in the solar wind. In the limit of a planar sheath in front of the instrument aperture, the positively charged spacecraft attracts the electrons and changes their initial trajectories. The focusing affect alters the solid angle viewed by the instrument, thus altering the geometric factor needed to convert instrument counts to the electron distribution function. The effective geometric factor is thus not constant, but dependent on the electron energy and local plasma density. Scime et al. ${ }^{?}$ generalized their correction model for sheaths of arbitrary, but known, shapes and demonstrated effective measurement correction for low energy plasma measurements for the Ulysses and IMP spacecraft if the local plasma density were known 
through other measurements. By flying TECHS and a GNSS receiver together on CuSPED, a low-risk, low-power spacecraft charging calibration technique will be validated for use on CubeSats.

\section{B. GNSS Atmospheric Sounding}

Previous LEO satellite missions have demonstrated an electron density imaging capability within the spacecraft orbital plane by processing raw GNSS observables collected from an antenna that is pointing in the spacecraft zenith direction for a receiver that is also used as the primary navigation receiver? ? This technique, applied on the CHAMP spacecraft, was validated both by correlating electron density estimates with the measurements obtained in-situ by an on-board Langmuir probe citeheise2002sounding and more recently by comparing them with coincident measurements obtained by the IMAGE spacecraft. ? To achieve this capability, dual-frequency GPS carrier-phase observables were tracked by JPL's software defined 'BlackJack' receiver, precise orbit determination of the CHAMP spacecraft was used to calibrate the raw observables such that slant Total Electron Content (TEC) could be extracted, and upward looking TEC values were assimilated into an a priori ionosphere model based on ground based tracking stations.

By leveraging NASA software tools such as JPL's GIPSY-OAISIS and JPL/USC's Global Assimilative Ionospheric Model (GAIM) ${ }^{\text {? }}$ CuSPED's raw GNSS measurements will be processed to yield high-precision electron density profiles. GIPSY-OASIS is a software library of precise orbit determination tools that includes many high-fidelity models for Earth orbiting spacecraft and features a state-of-the-art technique for fixing GPS carrier-phase biases.? GIPSY-OASIS has been used as NASA's primary precise orbit determination software for many LEO missions, including CHAMP,? GRACE, ${ }^{?}$ and Jason-1, ${ }^{?}$ amongst others. Under this award, a CubeSat specific set of GIPSY model configurations will be designed and evaluated. GAIM is a physics-based 3D data assimilation model that uses both 4DVAR and Kalman filter techniques to solve for the ion and electron density states and key drivers such as equatorial electrodynamics, neutral winds, and production terms. GAIM can accept as input ground GPS TEC data from 1000+ sites, occultation links from CHAMP, SAC-C, and the COSMIC constellation, UV limb and nadir scans from the TIMED and DMSP satellites, and in situ data from a variety of satellites (e.g., DMSP and C/NOFS). CuSPED's GNSS instrument will obtain important data for understanding the dynamics and coupling of the atmosphere and will provide data for configuring NASA's existing GNSS processing software for CubeSat applications.

\section{Mission Implementation}

\section{A. Concept of Operations and Architecture}

The 3U CuSPED spacecraft will be built, functionally tested and environmentally tested at West Virginia University (WVU). To motivate discussion of the CuSPED flight system, instruments, ground software and telecommunications, the envisioned mission architecture and operations concept is depicted in Figure ??. The remaining technical approach subsections of the proposal offer detailed descriptions about CuSPED's two instrument payloads, flight system, telecommunications subsystem, flight software architecture and integration test plans.

\section{B. TECHS Plasma Spectrometer Payload}

The Thermal Electron Capped Hemisphere Spectrometer (TECHS) is a miniature electrostatic analyzer designed specifically to measure the low-energy $(0.3-100 \mathrm{eV})$ electron plasma distribution of Earth's ionosphere. The TECHS instrument consists of six major components: ${ }^{\text {? }}$ a top hat electrostatic analyzer to provide energy analysis and particle arrival pitch angle information; a microchannel plate (MCP) stack to provide electron multiplication; a discrete anode imaging system allowing for simultaneous measurement of multiple pitch angle bins; an active aperture biasing system to mitigate repulsive spacecraft charging affects; and UV absorptive coatings to eliminate solar photon induced spurious events. The instrument sensor is approximately the size of a $35 \mathrm{~mm}$ film container (i.e. $50.8 \mathrm{~mm}$ tall and $32 \mathrm{~mm}$ diameter). The instrument has a cylindrically symmetric 360 azimuthal field of view. Only charged particles for which the electrostatic force balances the centripetal force will travel through the selection gap and be detected. Multiplication of the signal occurs in a 'Z-stack' microchannel plate electron multiplier which is then imaged by discrete anode detectors.

TECHS was originally flown on the Sounding of the Cleft ion Fountain Energization Region (SCIFER) 


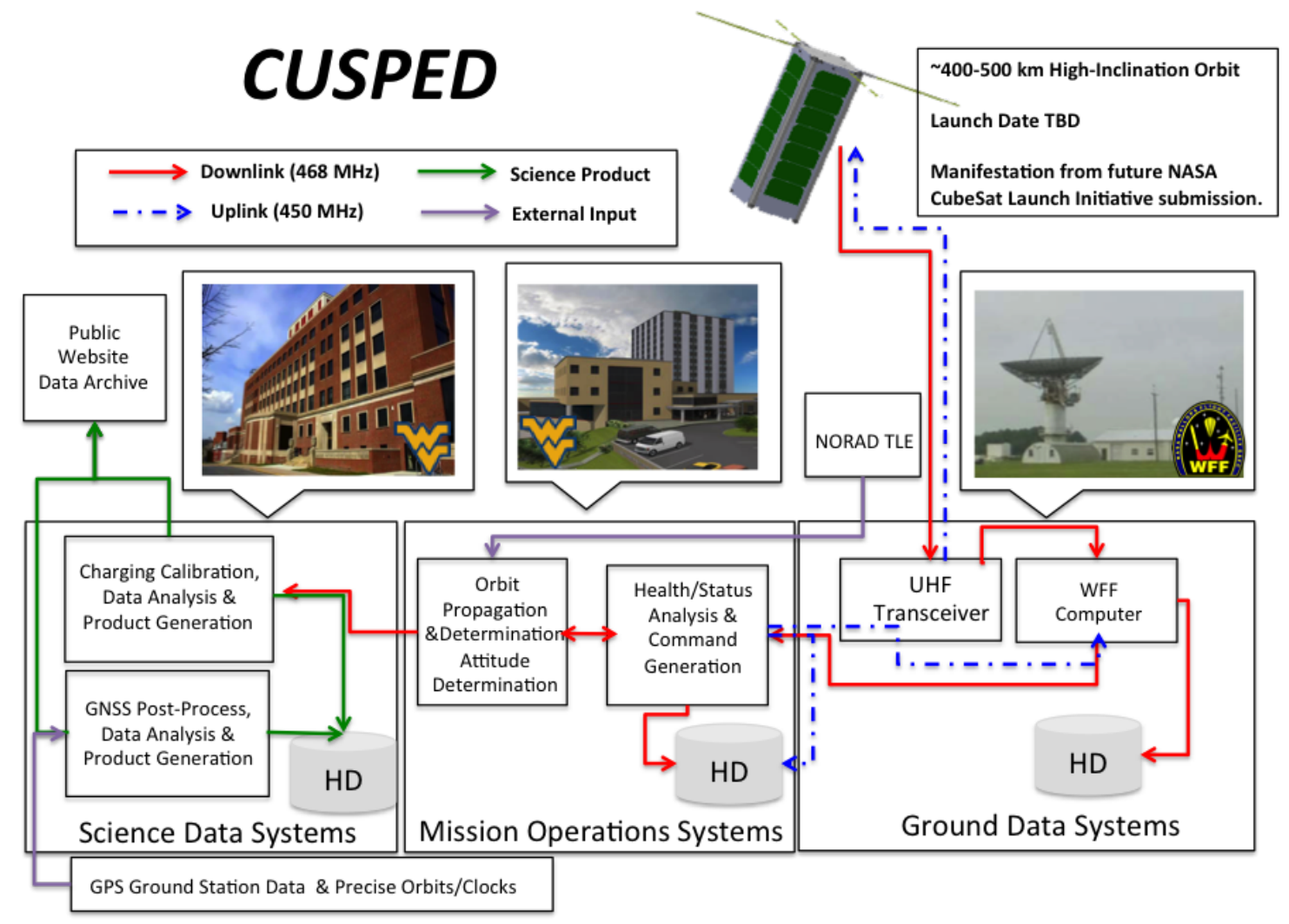

Figure 1. CuSPED's envisioned architecture and operations concept. A high-inclination LEO orbit will allow for passes through the auroral region. Command planning and engineering data analysis will be executed at WVU's engineering building. Telemetry will be handled through NASA's UHF antenna at WFF. Science products will be developed at WVU and archived on a public website.

sounding rocket in 1995? Analysis of the measured electron phase space density yielded increased electron temperatures in the cleft region associated with significant ion outflow. TECHS and TICHS instruments were also flown on the CAPER sounding rocket in 1999.?

\section{FOTON GNSS Receiver Payload}

Originally developed as a ground-based science-grade software receiver for monitoring ionospheric scintillation and total electron content (TEC), the Fast, On-Orbit TEC, Observables, Navigation (FOTON) ${ }^{\text {(Figure }}$ ??) was designed to provide high-quality GNSS signal observations in space. The receiver hardware was miniaturized and the software has been adapted for LEO operations. FOTON now fits within a $0.5 \mathrm{U}$ CubeSat form factor $(8.3 \times 9.6 \times 3.8 \mathrm{~cm})$, weighs less than $400 \mathrm{~g}$, and consumes $4.5 \mathrm{~W}$ of instantaneous power, which can be reduced to $<1 \mathrm{~W}$ orbit average power with efficient on-off duty cycling. A 2012 sounding rocket flight demonstration and extensive testing performed within the past year demonstrates that FOTON is a robust, versatile, high-precision dual-frequency space receiver. Its low cost, size, weight, and power are key enablers for future small-satellite missions.

For CuSPED, a new release of the FOTON hardware will be developed to fit within a smaller PC-104 standard form factor for ease of installation on a CubeSat. Further, FOTON software will be modified to support mission requirements, and performance will be validated on a real-time GPS signal simulator at Georgia Tech. Georgia Tech will be responsible for modification, testing, and delivery of the flight unit FOTON GNSS receiver and will provide technical support during the integration and testing phase of the project. The WVU team will be responsible for the FOTON's integration and onboard real-time software interfacing within CUSPED. 


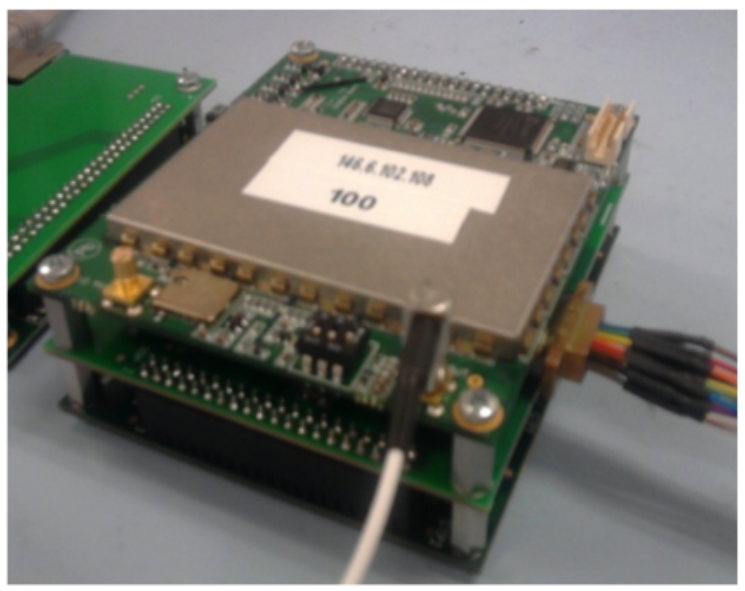

Figure 2. FOTON GNSS Receiver. FOTON will be miniaturized into the PC-104 form factor for integration on CuSPED.

\section{GNSS Data Processing Software}

CuSPED's GNSS data processing will leverage NASA's GNSS-Inferred Positioning System and Orbit Analysis Simulation Software (GIPSY-OASIS), NASA JPL's Global Ionospheric Model (GIM), and JPL/USC's Global Assimilative Ionospheric Model (GAIM). First, CuSPED's precise orbit will be determined by employing GIPSY-OASIS using a dynamic model customized for CUSPED. Next, CUSPED's raw GPS phaseleveled line-of-sight TEC observables will be corrected for satellite and receiver differential delays and hardware biases using GIM. Finally, the resulting absolute slant GPS-derived TEC observables from CuSPED will be used as input to GAIM along with the TEC links from approximately 200 ground-based geodeticprecision GPS receivers (e.g. International GNSS Service sites). By assimilating both the ground-based and space-based CuSPED GPS data into GAIM, 3D electron densities with voxels up to $2000 \mathrm{~km}$ altitude will be recovered. Because ground-based GPS data cannot perform well in providing vertical resolution of electron density profiles, CuSPED GPS measurements will be critical in improving vertical resolution above the F2 peak layer. CuSPED's science products will include 2D and 3D electron densities for various geographic regions that can be validated using Jason-derived vertical TEC as well as incoherent backscatter radar measurements.

\section{E. Telecommunications Architecture}

As depicted in Figure ??, CuSPED's baseline telecommunications operations plan is to utilize NASA's Wallops Flight Facility (WFF) UHF antenna for $468 \mathrm{MHz}$ downlink and $450 \mathrm{MHz}$ uplink. This configuration has the advantages of: 1) proven heritage, ${ }^{?}$ 2) high-throughput, and 3) WVU in-house experience through our participation in the on-going NASA IVV STF-1 CubeSat mission which is using the same set-up.

An L3 Cadet NanoSat transceiver (468 MHz downlink, $450 \mathrm{MHz}$ uplink) with a deployable UHF dipole antenna will be included in the flight system design to communicate with WFF's 18.3-meter UHF ground antenna outfitted with a software defined radio. WVU will seek an NTIA transmission license for the 468 $\mathrm{MHz}$ band, a process that is expected to take around a year and has already successfully been granted for other CubeSats missions (i.e. DICE/DICE II, MicroMAS). $450 \mathrm{MHz}$ is already designated for the purposes of Earth to space satellite teleoperation. Assuming a nominal design orbit of 72 degrees inclination and $500 \mathrm{~km}$ altitude, a link budget was derived using the Amateur SATellite (AMSAT)/ International Amateur Radio Union's annotated link budget tool and it indicates sufficient margins are achieved for the downlink and uplink assuming respective data rates of $1.5 \mathrm{Mbps}$ and $38.4 \mathrm{kbps}$.

\section{F. Flight System Architecture}

With the exception of the TECHS and FOTON instruments, CuSPED's remaining flight system elements will be comprised of COTS CubeSat subsystems that have high flight-heritage to minimize risk. Figure ?? details CuSPED's overall system design in terms of selected subsystem elements, subsystem vendors, 
required interfaces (power and data protocols), as well as the breakdown of partner responsibilities on the mission.

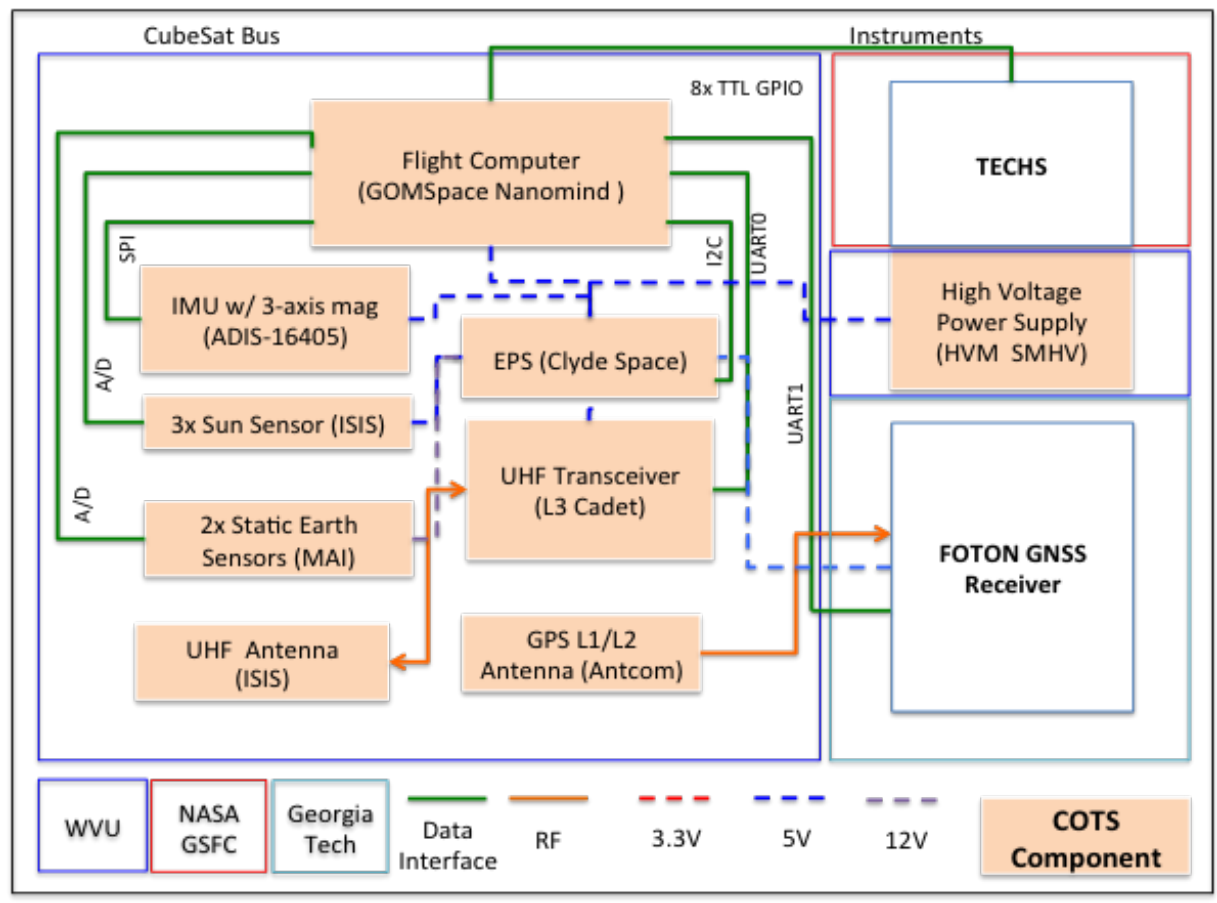

Figure 3. CuSPED's high-level system design, flight elements and required interfaces.

Two custom printed circuit boards (PCB) will be developed at WVU to interface COTS subsystems with the flight computer, one PCB for the attitude determination sensors including a set of tri-axial Micro-ElectroMechanical System (MEMS) inertial measurement units and magnetometers, and one PCB for TECHS high-voltage power supply, which is a COTS component that has flight heritage from NSF/NASA Firefly Mission.

Table ?? highlights top-level flight system margins for key CubeSat system design parameters including Current Best Estimates (CBE) for mass, volume, and data capacity. The overall volume allocation has little uncertainty for the COTS subsystem components and the FOTON instrument, leaving ample margin for TECHS. The power needed during science collection is $6 \mathrm{~W}$ (without duty cycling FOTON). The current best estimate plus contingency ( $30 \%$ for all non-COTS components) of the overall flight system mass is 3.74 $\mathrm{kg}$, leaving $7 \%$ margin.

Table 1. Top-Level Design Margins

\begin{tabular}{rrrr} 
Parameter & Requirement & CBE & Margin \\
\hline Mass & $<4.00 \mathrm{~kg}$ & $3.74 \mathrm{Kg}$ & $7 \%$ \\
Volume & $3 \mathrm{U}$ & $2.5 \mathrm{U}$ & $16 \%$ \\
Downlink Link Margin & EbN0 9.5 dB & $19.1 \mathrm{~dB}$ & $50 \%$ \\
Downlink Throughput & $56 \mathrm{MB} /$ pass & $90 \mathrm{MB} /$ pass & $37 \%$
\end{tabular}

CuSPED's envisioned 3U volume configuration is depicted in Figure ?? with each component labeled. An Antcomm dual-frequency GNSS antenna will occupy a 1U face. Earth and Sun sensors will provide coarse attitude knowledge and magnetorquers that are embedded in the solar panels will be used to periodically orient the GPS antenna anti-Earthward. Approximate subsystem thermal properties will be added to the CAD model, should it be needed, active thermal management will be performed by duty cycling the on-board telemetry radio. 


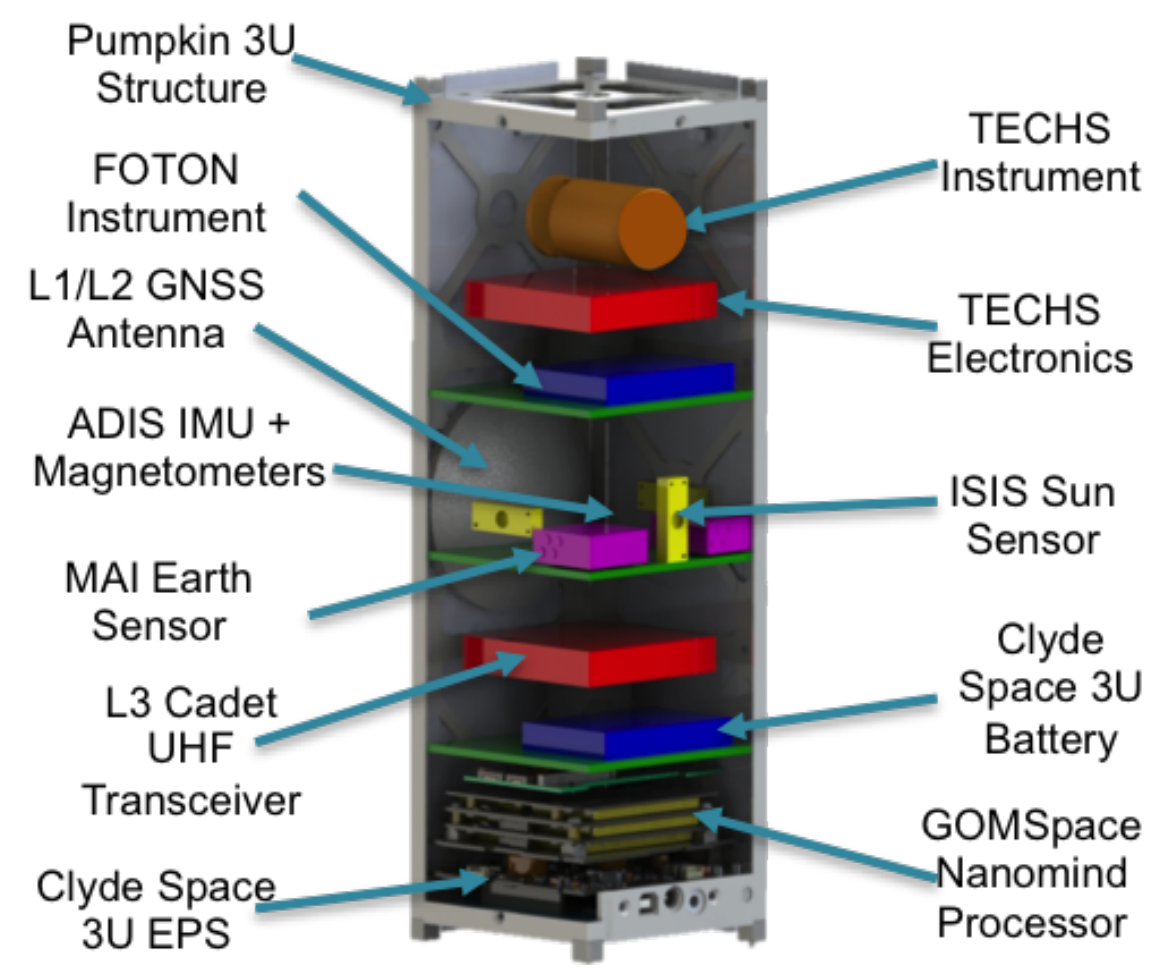

Figure 4. CuSPED's 3U Component Layout

\section{G. Flight Software}

CuSPED's flight software will collect, store, and telemeter instrument data and schedule each CubeSat subsystem based on a set of operational modes designed by the WVU team. Figure ?? indicates each subsystem's communication protocol (UART, SPI, A/D, etc.) for which WVU will develop and test low-level software applications for both the science instruments and the COTS subsystems (Electronic Power Supply, Inertial Sensors, etc.) to interface with the flight computer. To handle on-board operational scheduling and prioritization, CUSPED will leverage re-use from an on-going $3 \mathrm{U}$ CubeSat mission being led by NASA IVV that is named STF-1. A main objective of STF-1 is to develop a Core Flight Executive (CFE) Software Bus for Commands Data Handling on CubeSat missions. To leverage this software approach, CUSPED has selected the GOMSpace Nanomind Flight CPU, and L3 Cadet radio, which is being used for STF-1 as well as the GSFC Dellngr mission.

\section{H. Assembly Integration and Test}

During the first period of CuSPED's development phase, all flight system COTS components will be integrated in a 'flat-sat' configuration at WVU to allow for flight software development and debugging without need for mechanical interfaces. In parallel, the custom interface PCB's will be designed and assembled. The telemetry subsystem will be tested end-to-end during a site visit to NASA GSFC Wallops Flight Facility, and the removal of the FOTON's payload COCOM limits (i.e., velocity and altitude restrictions) will be verified during its testing on a GPS constellation simulator at Georgia Tech.

During and after fabrication at GSFC, the TECHS instrument will be tested and calibrated in the GSFC electron beam calibration facility. The GSFC electron beam calibration facility consists of two identical oil-free high vacuum chambers surrounded by an array of Helmholtz coils to mitigate the effects of the local geomagnetic field. Each chamber is equipped with a Kimball Physics Model electron source and an articulating instrument mount table with four degrees of freedom After 18-months, the TECHS instrument will be delivered from NASA GSFC to WVU for additional testing in the WVU Space Simulation Chamber.

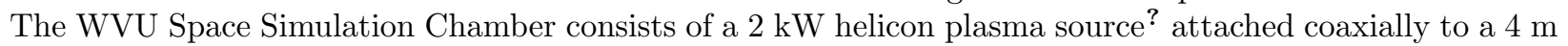


long, $2 \mathrm{~m}$ diameter aluminum vacuum chamber (Figure ??). The entire chamber is an oil free system suitable for the testing of space plasma instruments containing microchannel plates. For the purposes of testing the TECHS instrument, the plasma source will be operated at the lowest feasible neutral gas pressures to create plasma in the space simulation chamber with densities on the order of $10^{8} \mathrm{~cm}^{-3}$. After testing in the WVU Space Simulation Chamber, the TECHS instrument and electronics will be mounted into CuSPED.

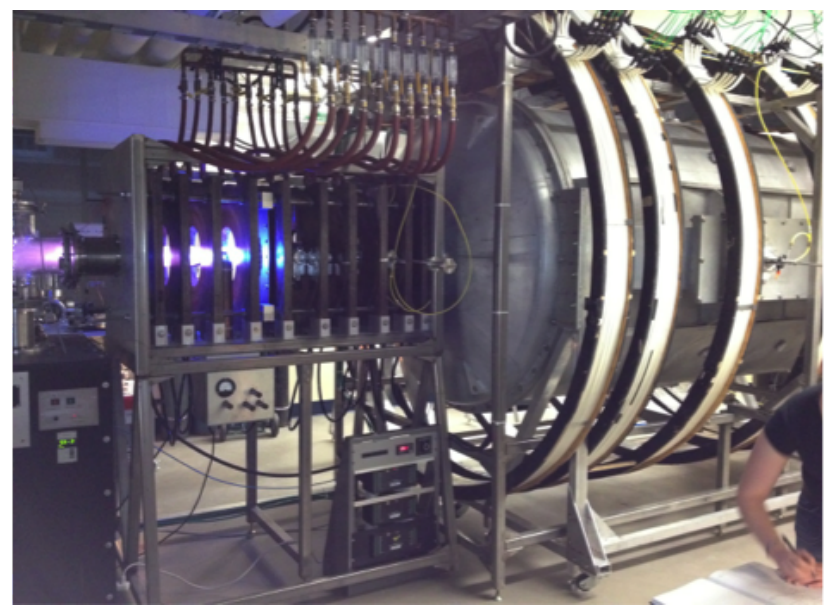

Figure 5. The WVU Space Plasma Simulation Chamber will be used for testing of the TECHS instrument.

\section{Mission Operations}

As depicted in the envisioned operations concept (Figure ??), once CuSPED's data has been telemetered NASA WFF's ground station, it will be transferred to the engineering operations and science teams at WVU via the Internet. Engineering operations will consist of monitoring the number of health and status information that will be included in the telemetry and then subsequently determining the operational modes to send to CUSPED during the next uplink. GPS orbital data will be used to seed the prediction for the next data pass window, and NORAD TLEs will be used as a back up. Science operations will initially be split between the two instruments and will yield two independent products for comparison. The science data from the FOTON instrument will consist of raw dual-frequency GNSS psuedorange and carrier-phase measurements. From these data, the low-level science products derived will include the precise orbit estimates and ionospheric TEC estimates. These products will then feed into GAIM for the generation of electron density profiles, which will be constrained around CUSPED's orbital path by the data. The external data required for the GNSS science operations are GNSS precise orbit and clocks and ground station tracking data, both of which are made freely available by the International GNSS Service. The science data from the TECHS instrument will consist of particle precipitation counts. TECHS data will be combined with the precise orbit estimates to map the particle environment as a function of orbital position. This will enable the validation of charging calibration procedure detailed earlier. In addition, TECHS electron precipitation measurements will be directly comparable with the GNSS derived profiles, enabling cross-validation of the two approaches.

\section{Conclusion}

This paper provided an overview including details of the mission design, in terms of its science motivation, operations concept, payload description, flight system architecture, telecommunications architecture and flight software for a space weather focused $3 \mathrm{U}$ CubeSat mission that has been proposed for implementation by West Virginia University in collaboration with Georgia Tech, NASA GSFC and NASA JPL. The CuSPED will fly TECHS, a small plasma spectrometer, alongside FOTON, a small space-capable GNSS receiver for the purpose of profiling the upper ionosphere's electron density along the spacecraft's trajectory. Further, by collocating these two instruments, CuSPED will provide unique data set for validating a spacecraft charging calibration approach that is applicable for use on other CubeSat platform's and mission concepts. 


\section{Acknowledgments}

The authors wish to thank the West Virginia Space Grant Consortium for supporting the development of this mission concept. 